\title{
ACHIKO-M Database for High Myopia Analysis and Its Evaluation
}

\author{
Fengshou Yin, Ruoying Li, Zhuo Zhang, Jun Cheng, Yanwu Xu, Damon Wee Kee \\ Wong, Ngan Meng Tan, Ying Quan, Ai Ping Yow, Gopalakrishnan Kavitha, Guozhen \\ $\mathrm{Xu}$, and Jiang Liu \\ Institute for Infocomm Research, Agency for Science, Technology and Research, Singapore
}

\begin{abstract}
Myopia is the leading public health concern with high prevalence in developed countries. In this paper, we present the ACHIKO-M fundus image database with both myopic and emmetropic cases for high myopia study. The database contains 705 myopic subjects and 151 normal subjects with both left eye and right eye images for each subject. In addition, various clinical data is also available, allowing correlation study of different risk factors. We evaluated two state-of-the-art automated myopia detection algorithms on this database to show how it can be used. Both methods achieve more than $90 \%$ accuracy for myopia diagnosis. We will also discuss how ACHIKO-M can be a good database for both scientific and clinical research of myopia.
\end{abstract}

\section{$1 \quad$ Introduction}

High myopia is a type of severe shortsightedness characterized by the growing axial length of the eye. People with high myopia usually have a spherical equivalent refraction of a least -6 diopers [1]. Over the last few decades, high myopia has increased in prevalence across populations and shifted to a younger age [2]. High myopia can lead to a number of pathologic complications such as posterior vitreous detachment, retinal detachment and pathological myopia, if degenerative in nature. Peripapillary atrophy (PPA), which is a recession of retina tissues, is an important risk factor and indicator of myopia [3]. In fundus images, PPA is a crescent-shaped area adjacent or around the optic disc (Fig. 1). It is found that the area of PPA is strongly correlated with the severity of myopia [4]. Thus, identifying PPA from fundus images is essential to help clinicians in the diagnosis and treatment of high myopia.

To provide a fast and accurate way of detecting PPA and hence pathological myopia or high myopia, researchers have developed several automated systems using advanced image processing and machine learning techniques. A segmentation based approach is proposed in [5] as part of the PAMELA (Pathological Myopia Detection Through Peripapillary Atrophy) system. Two different settings of the variational level method are employed to segment the optic disc region as well as a larger region, including optic disc and possible PPA. After computing the difference of the two re- 
gions, the presence of PPA is determined by the asymmetry of the difference in the temporal and nasal sides. In [6], a similar idea is adopted with the difference of segmenting the two regions using a Chan-Vese model with shape constraints. Experimental results show that this method can repeatedly detect both the sizes of the OD and PPA region automatically, and achieved a mean accuracy of $91.3 \%$ and $92.5 \%$ in defining the size of the OD and PPA respectively. In [7], a texture analysis based approach was proposed. After automatic detection of the region of interest and optic disc, texture-based metrics are generated, categorized and grouped into zones for generation of features. Finally, these sector-based texture features will be used in a support vector machine to determine the presence of PPA, and correspondingly pathological myopia. Recently, much effort has been spent in pathological myopia detection through machine learning techniques $[8,9,10]$. In [8], biologically inspired features (BIF) from a focal region of the retinal image are used for the automatic detection of PPA. As BIF is an intrinsically low dimensional feature embedded in a high dimensional space, the authors proposed a manifold learning method to reduce the feature dimension of BIF, which achieved better performance. The BIF based approach was tested on a total of 1584 images from SCORM, and the results achieved an accuracy of more than $90 \%$. In [9], a bag-of-feature and sparse learning based framework was proposed for the automatic recognition of pathological myopia, where the most related visual features are discovered via sparse learning concurrently. Experimental results based on the evaluation on a population based dataset of $2258 \mathrm{im}$ ages achieved an area under curve (AUC) of $0.964 \pm 0.007$ and $90.6 \pm 0.1 \%$ balanced accuracy with $85 \%$ specificity. In [10], another computer-aided diagnosis framework called Pathological Myopia diagnosis through Biomedical and Image Informatics (PM-BMII) was proposed to intelligently fuse heterogeneous biomedical information to improve the accuracy of disease diagnosis using multiple kernel learning methods. The proposed PM-BMII framework has been tested on the Singapore Malay Eye Study (SiMES), and the performance can reach an AUC of around $88 \%$.

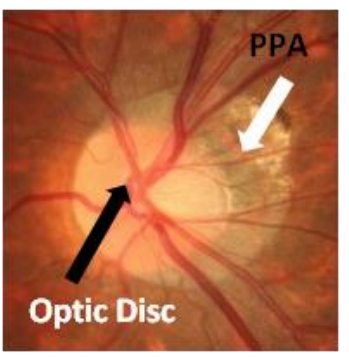

(a)

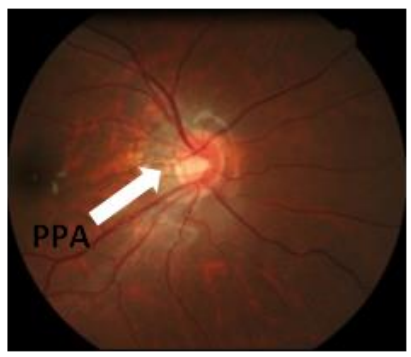

(b)

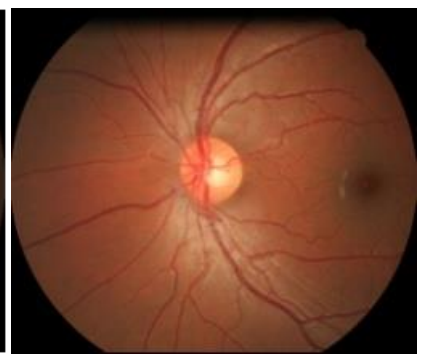

(c)

Fig. 1. Sample fundus images with PPA (a, b) and without PPA (c).

In this paper, we present a specially designed retinal fundus image database for high myopia study. The database is constructed from a case-controlled study that contains only normal subjects and high myopia subjects. The rest of the paper is organized as follows. In Section 2, we introduce the data available in the ACHIKO-M database and 
how it can be used in myopia study. Section 3 reviews two state-of-the-art pathological myopia detection methods $[8,9]$ in more details, and compares the experimental results from both methods on this database. We conclude this paper with discussions and conclusions in the last section.

\section{ACHIKO-M Database}

\subsection{Data Source}

The ACHIKO-M database is constructed from a high myopia study on soldiers in Singapore Armed Forces from 2011 to 2012. A total of 1712 images from 856 subjects aged from 19 to 25 are selected with one optic disc centered image for each eye. The images have the same dimension of 3504 x 2336 pixels, and are of JPEG format. All images are anonymized to hide critical information of the subjects. Within the database, 705 subjects are diagnosed as high myopic and the rest 151 subjects are emmetropic (normal vision).

Table 1. SELECTED CLINICAL DATA OF ACHIKO-M DATDABASE

\begin{tabular}{lc}
\hline \multicolumn{1}{c}{ Parameter } & Details \\
\hline SE & Spherical Equivalent \\
SPH & Sphere (SPH lens power) \\
Age & Cylinder (CYL astigmatism) \\
AgeRefractiveError & Age when examination \\
AgeWearGlass & Age when SE reach -6D \\
Gender & Age when start wearing glasses \\
Ethnic Group & Male or female \\
Eye Surgery & Chinese, Malay, Indian or others. \\
Cornea & Eye surgery other than laser vision correction \\
Lens & Normal or abnormal \\
Pupil & Normal or abnormal \\
Conjuctiva & Normal or abnormal \\
Anterior Chamber & Normal or abnormal \\
Comments & Normal or abnormal \\
\hline
\end{tabular}

\subsection{Clinical Data}

Besides 2D retinal fundus images, various clinical information is available for all subjects. There are four categories of clinical information: demographic data, ocular history, refractive status and anterior segment examination data. Table 1 shows some 
important parameters available in the clinical data. In all subjects, 92\% are Chinese, $5 \%$ are Malay, and 3\% are Indian and others.

One of the most important parameters is the spherical equivalent (SE), which has a direct effect on how the fundus image looks like. The distribution of this parameter is shown in Fig. 2. We can see that all myopic eyes have SE of at least -6 D, while emmetropic eyes have SE values between -0.5 D to 0.5 D. Thus, ACHIKO-M is a strictly controlled database that can be used to study obvious differences between myopic and emmetropic cases.

The distributions of age when myopic subjects start wearing glasses and age when their SE values reach $-6 \mathrm{D}$ are shown in Fig. 3. The average age for the subjects to start wearing glasses is 7.57 , with $90.6 \%$ of them start before the age of 10 . In addition, the average for SE to reach $-6 \mathrm{D}$ is 13.71 .

\subsection{Proposed Usage}

The database can be used in several ways, both scientifically and clinically:

a) To develop automated high myopia detection algorithms;

b) To study correlation of PPA size and spherical equivalent;

c) To develop machine learning and regression models for predicting SE from fundus images;

d) To benchmark automated myopia detection algorithms;

e) To clinically analyze correlation of high myopia with other risk factors such as disc tilting.

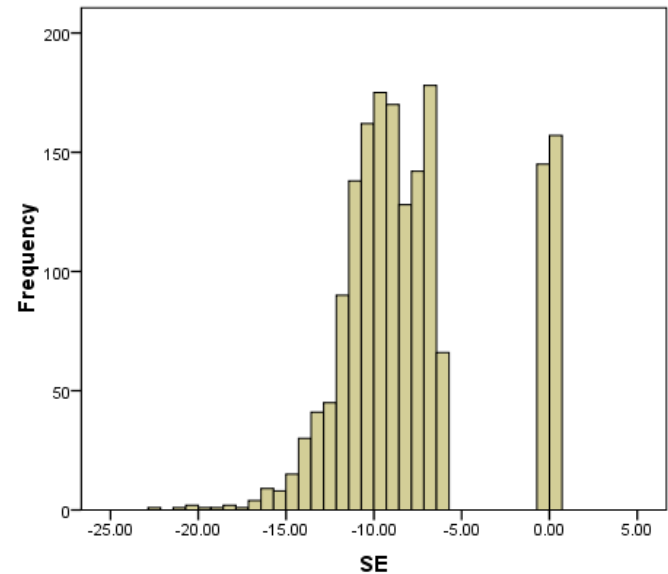

Fig. 2. Distribution of spherical equivalent for ACHIKO-M database (Frequency based on number of eyes). 


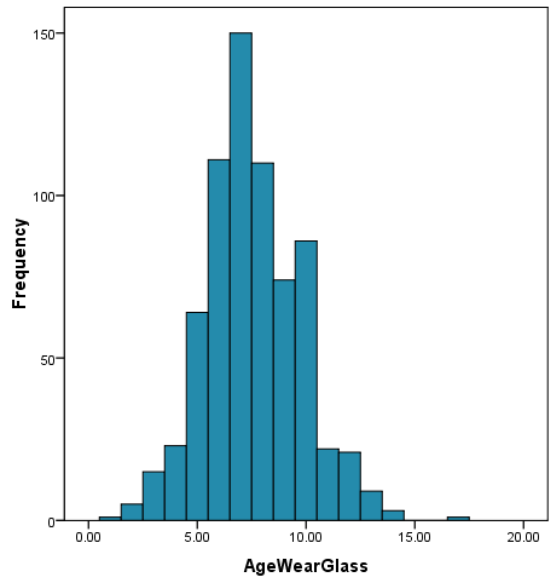

(a)

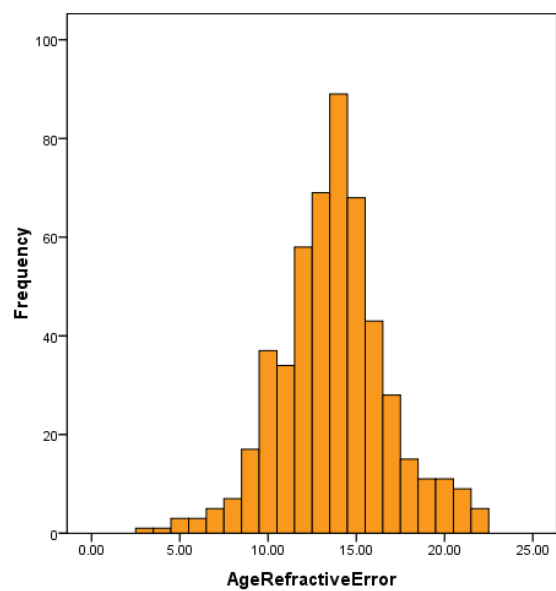

(b)

Fig. 3. Distribution of AgeWearGlass and AgeRefractiveError for ACHIKO-M database (Frequency based on number of subjects).

\section{Experiments}

\subsection{Experiment Settings}

In order to do a holistic analysis of both systems, we performed a 2-fold cross validation. For the dataset, half of the myopic cases and half of the control cases are randomly selected for training $\left(\mathrm{S}_{0}\right)$. The rest of the cases are used for testing $\left(\mathrm{S}_{1}\right)$. Then, the training and testing datasets are switched and the average result of the two splits is reported. This experiment setting has the advantage of ensuring a large dataset for both training and testing. Moreover, each sample is used for both training and testing on each fold. The process is repeated for ten times.

\subsection{Sparse Biologically Inspired Feature Manifold (SBIFM)}

In [8], the optic disc is first located through an adaptive thresholding and constrained elliptical Hough Transform. The focal region of interest around the optic disc is then defined as an ellipse with the same centre and angle with the optic disc, but larger radii. The rationale for this step is to find a region that includes PPA and excludes other regions as much as possible.

Biologically inspired feature (BIF), which consists of 34 feature maps from intensity units, color units and $\mathrm{C} 1$ units, is extracted to mimic ophthalmologists' visual cortex to recognize PPA. As BIF is a low dimensional feature artificially embedded in a high dimensional space, redundancy reduction is performed through sparse transfer learning. The selective pair-wise discriminant analysis is used to formulate the sparse transfer learning. Finally, the processed features are passed to a support vector machine to train the classification model and perform testing. 


\subsection{Bag-of-Feature and Sparse Learning (BoFSL)}

In [9], a machine learning based framework was proposed to learn the visual correspondence to myopia. In the learning phase, a codebook is generated using bag-offeature model and sparse learning feature selection. The classification model is then learnt.

To build the bag-of-feature model, SIFT (Scale-invariant feature transform) features are extracted from the green channel image by Harris-Laplacian, HessianLaplacian and Maximally Stable Extremal Region detectors. These features, each represented as a quantized 128-dimensional histogram, include both corner and blob information. Finally, a k-means clustering is used to generate the codebook.

To obtain higher precision and speed by using only relevant information, feature selection is necessary. Denoting the original feature as $\mathbf{f}_{\mathrm{i}}$, which consists of $g$ groups, and its label as $d_{i} \in\{-1,+1\}$, the feature selection can be done by minimizing the following objective function:

$$
\min _{\omega} \sum_{i=1}^{n}\left\|d_{i}-\omega^{T} \mathbf{f}_{i}\right\|^{2}+\lambda_{1}\|\omega\|+\lambda_{2} \sum_{j=1}^{g}\left\|\omega_{j}\right\|_{2}
$$

where $n$ is number of training samples, $\omega$ is the weight to be learnt, $\lambda_{1}$ and $\lambda_{2}$ are parameters to control sparsity. In this equation, the first term represents the label estimation error, the second term is a $L_{1}$ norm based regularizer to enforce feature sparsity and the last term is a $L_{1,2}$ norm based regularizer to enforce group sparsity.

\subsection{Evaluation Metric}

To evaluate the classification performance of both methods, we use the accuracy $(A C C)$ metric which can be calculated from sensitivity $(S E N)$ and specificity $(S P E)$ :

$$
\begin{gathered}
A C C=S E N * \text { Pre }+S P E *(1-\text { Pre }) \\
S E N=\frac{T P}{T P+F N} \\
S P E=\frac{T N}{T N+F P}
\end{gathered}
$$

where $T P, T N, F P$ and $F N$ denote the number of true positives, true negatives, false positives and false negatives respectively, and Pre is the prevalence of the disease in the dataset.

\subsection{Experimental Results}

The classification accuracy for methods in [8] and [9] is shown in Table 2. We can see that these two methods achieve very close performance, with accuracy of more than $90 \%$. The results show that both methods are able to differentiate high myopia from normal with a high accuracy. At the same time, it also demonstrates that 
ACHIKO-M is a good database for developing and benchmarking automatic myopia detection systems.

Table 2. Comparison of classification accuracy for sparse biologically inspired manifold (SBIFM) and bag-of-feature and sparse learning (BoFSL) on ACHIKO-M database.

\begin{tabular}{|c|c|c|}
\hline Round & SBIFM & BoFSL \\
\hline 1 & 0.966 & 0.957 \\
\hline 2 & 0.959 & 0.946 \\
\hline 3 & 0.971 & 0.950 \\
\hline 4 & 0.962 & 0.949 \\
\hline 5 & 0.972 & 0.952 \\
\hline 6 & 0.970 & 0.946 \\
\hline 7 & 0.959 & 0.949 \\
\hline 8 & 0.972 & 0.946 \\
\hline 9 & 0.964 & 0.957 \\
\hline 10 & 0.960 & 0.960 \\
\hline Average & $\mathbf{0 . 9 6 6}$ & $\mathbf{0 . 9 5 1}$ \\
\hline
\end{tabular}

\section{Conclusions}

In this paper, we present the ACHIKO-M retinal fundus image database for high myopia study. The database contains both high myopic cases and control cases, making it ideal for developing and benchmarking computer-aided diagnosis systems. We also compared two state-of-the-art computerized pathological myopia detection systems and tested them on the ACHIKO-M database. Results show that both systems perform well on this database, demonstrating its usefulness in high myopia study. Future work includes annotating the exact location of the optic disc and PPA for a subset of the database to study the correlation of PPA size and severity of myopia, as well as developing PPA segmentation algorithms.

\section{References}

1. Shih, Y.F., Ho, T.C., Hsiao, C.K., Lin, L.L.: Visual outcomes for high myopic patients with or without myopic maculopathy: a 10 year follow up study. Br J Ophthalmol, vol. 90, no. 5, pp.546-50 (2006)

2. Jones, D., Luensmann, D.: The prevalence and impact of high myopia. Eye Contact Lens 38(3): 188-196 (2012)

3. Hayashi, K., Ohno-Matsui, K., Shimada, N., Moriyama, M., Kojima, A., Hayashi, W., Yasuzumi, K., Nagaoka, N., Saka, N., Yoshida, T., Tokoro, T., Mochizuki, M.: Long-term pattern of progression of myopic maculopathy: A natural history study. Ophthalmology, vol. 117, pp. 1595-1611 (2010) 
4. Hyung, S. M., Kim, D.M., Hong, C., Youn, D.H.: Optic disc of the myopic eye: Relationship between refractive errors and morphometric characteristics. J. Ophthalmol., vol. 6, pp. 32-35 (1992)

5. Tan, N.M., Liu, J., Wong, D.W.K., Lim, J.H., Zhang, Z., Lu, S., Li, H., Saw, S.M., Wong, T.Y., Tong, L.: Automatic detection of pathological myopia using varitional level set. In: Int. Conf. IEEE Eng. Med. Bio. Soc, pp. 3609-3612. Minnesota (2009)

6. Lu, C.K., Tang, T.B., Murray, A.F., Laude, A., Dhillon, B.: Automatic parapapillary atrophy shape detection and quantification in colour fundus images. In: IEEE Conf. Biomed. Circuits Syst, pp. 86-89. Paphos (2010)

7. Liu, J, Wong, D.W.K., Lim, J.H., Tan, N.M., Zhang, Z., Li, H., Yin, F., Lee, B.H., Saw, S.M., Tong, L., Wong, T.Y.: Detection of pathological myopia by Pamela with texturebased features through an SVM approach. J. Healthcare Eng., vol. 1, no. 1, pp. 1-11 (2010)

8. Cheng, J., Tao, D.C., Liu, J., Wong, D.W.K., Tan, N.M., Wong, T.Y., Saw, S.M.: Peripapillary Atrophy Detection by Sparse Biologically Inspired Feature Manifold. IEEE Transactions on Medical Imaging (TMI). Volume: 31, Issue: 12, Pages: 2355 - 2365 (2012)

9. Xu, Y., Liu, J., Zhang, Z., Tan, N.M., Wong, D.W.K., Saw, S.M., Wong, T.Y.: Learn to Recognize Pathological Myopia in Fundus Images Using Bag-of-feature and Sparse Learning Approach. In: IEEE 10th International Symposium on Biomedical Imaging (ISBI), pp. 888 - 891. San Francisco (2013)

10. Zhang, Z., Xu, Y., Liu, J., Wong, D.W.K., Kwoh, C.K., Shaw, S.M., Wong, T.Y.: Automatic Diagnosis of Pathological Myopia from Heterogeneous Biomedical Data. PLoS ONE, 8(6): e65736. doi:10.1371/journal.pone.0065736. (2013) 